\title{
Effects of long or short duration stimulus during high- intensity interval training on physical performance, energy intake, and body composition
}

\author{
Elaine Domingues Alves¹, Gabriela Pires Salermo', Valéria Leme Gonçalves Panissa² ${ }^{2}$ Emerson Franchini², Monica Yuri Takito ${ }^{1, *}$ \\ 'Department of Human Movement Pedagogy, School of Physical Education and Sport, University of São Paulo, São Paulo, Brazil \\ ${ }^{2}$ Department of Sport, School of Physical Education and Sport, University of São Paulo, São Paulo, Brazil
}

To compare the effects of 6 weeks of long or short high-intensity interval training (long- or short-HIIT) on body composition, hunger perception, food intake and rating of perceived exertion (RPE). Twenty previously untrained women ( $25 \pm 5$ years) were randomly assigned to do a longHIIT $(n=10)$ or a short-HIIT $(n=10)$. The long-HIIT group performed fifteen 1-min bouts at $90 \%$ of maximum heart rate (HRmax), interspersed by 30 -sec active recovery (60\% HRmax). The short-HIIT group performed forty-five 20 -sec bouts at $90 \%$ of HRmax, interspersed by 10 -sec active recovery $(60 \% \mathrm{HRmax})$. The training for both groups was conducted 3 times a week for 6 weeks. All subjects performed the Astrand cycle ergometer test to estimate maximal oxygen consumption $\left(\mathrm{VO}_{2 \max }\right)$ 1 week before and after the training period, as well as body composition,

\section{INTRODUCTION}

Physical inactivity and obesity with unfavorable outcomes for health (Blair and Church, 2004) support the recommendation for changes on the lifestyle that influence the body composition and health, such as eating habits improvement and inclusion of physical activity (Hauser et al., 2004; Rankin, 2015).

Among the strategies for fat mass (FM) reduction, recent studies suggest the high-intensity interval training (HIIT) (Airin et al., 2014; Gillen et al., 2016; Higgins et al., 2016; Macpherson et al., 2011; Trapp et al., 2008), which is characterized by successive periods of high-intensity effort interspersed with active or passive recovery, enabling higher workloads than in continuous exercise. Although the number of publications regarding this subject has increased, the regulation of HIIT has not been sufficiently studied which was estimated through circumferences and skinfold thicknesses. For all training sessions, the heart rate, visual scale of hunger, internal load, and RPE were recorded. In the first and last week of training, subjects were asked to record a 24-hr food diary for 3 days. Both training induced significant pre to post decreases for fat mass, fat percentage, waist circumference, sum of seven skinfolds and RPE. As expected estimated, the $\mathrm{VO}_{2 \max }$ increased in both groups. There were no differences for hunger perception, energy intake, and body mass. Long and shortHIIT resulted in fat loss, without altering the energy intake.

Keywords: Body composition, High-intensity interval training, Hunger, Energy intake
*Corresponding author: Monica Yuri Takito (iD https://orcid.org/0000-0003-0257-8765 Department of Human Movement Pedagogy, School of Physical Education and Sport, University of São Paulo (USP), Av. Prof. Mello Morais, 65, Butantã, São Paulo, SP 05508-900, Brazil

Tel: +55-11-3091-2306, E-mail: mytakito@gmail.com

Received: April 13, 2017 / Accepted: July 17, 2017 yet (Tschakert et al., 2015), due to the number of variables that can be manipulated, these variables namely: effort and pause intensity and duration, work: rest ratio, type of pause (active or passive), number and duration of series, duration and type of recovery between series. These combinations have been classified in four different high-intensity interval exercise (HIIE) models: (a) sprint interval training (SIT) - 30 sec of all-out effort for 2 to $4 \mathrm{~min}$ of pause; (b) repeated sprint training (RST) - 3 to $7 \mathrm{sec}$ of effort at around $160 \%$ of velocity associated with maximal oxygen consumption $\left(\mathrm{vVO}_{2 \max }\right)$ interspersed with recovery periods lower than $60 \mathrm{sec}$; (c) long-Interval HIIT - more than 60 sec of effort at around 100\%$120 \% \mathrm{vVO}_{2 \max }$; and (d) short-Interval HIIT - less than 60 sec of effort performed around $90 \%-100 \% \mathrm{vVO}_{2 \max }$. In this way, it is possible to potentiate the adaptive responses to the exercise with a great variety of protocols (Buchheit and Laursen, 2013). 
Recent studies showed the similar adaptations (Nalcakan, 2014) or even superior (Airin et al., 2014; Racil et al., 2013; Tremblay et al., 1994) when comparing HIIT with continuous training, showing to be a strong strategy to weight control and one of the factors that may influence changes in body composition is appetite suppression induced by HIIE (Panissa et al., 2016; Trapp et al., 2008). However, there is still no consensus regarding the best frequency, mode, intensity and duration (Gist et al., 2014).

Acute studies that maintain the effort-pause ratio constant (Islam et al., 2017; Price and Moss, 2007), but alter the duration of efforts, indicated that the physiological responses can be altered, affecting the use of substrates during the effort and resulting in different total energy expenditure. Indeed, Islam et al. (2017) demonstrated that shorter $(5 \mathrm{sec}: 40 \mathrm{sec})$ and medium $(15 \mathrm{sec}: 120$ sec) all-out efforts, in an effort-pause ratio of 1:8, resulted in higher energy expenditure during the session when compared to the traditional SIT protocol (30 sec:240 sec), although the longer induced higher fat oxidation postexercise when compared to the shorter protocol. Thus, these different physiological responses, repeated over time, could result in different chronic adaptations. Another consequence of this manipulation is the rating of perceived exertion (RPE), with studies showing that longer protocol results in larger values on the RPE (Price and Moss, 2007).

In the same way that manipulations can affect RPE, RPE can affect an individual's adherence to a regular exercise program (Price and Moss, 2007), the feelings that are induced by the physical exercise are related to the participation or not of regular physical exercise practice (Ekkekakis, 2009). Thus, if the feelings are negative, they may prevent the practice of the exercise, and, on the other hand, if the feelings are positive, they can promote the continued participation (Frazão et al., 2016; Garber et al., 2011). For the HIIT to be viable or sustainable, it is necessary evaluate the sensation it induces (Smith-Ryan, 2017).

Therefore, this research evaluates the chronic effect of varied duration of HIIE with the same effort-pause ratio on the body composition, aerobic power and energy intake. As a secondary objective, we evaluated the impact of such variation on RPE, hunger perception and food intake.

\section{MATERIALS AND METHODS}

\section{Design}

A quasi-experimental study was conducted with a nonequivalent control group design. The subjects were recruited through posters displayed in the vicinity. The identification of diseases and health problems, as well as the use of any drug or nutritional supplement were exclusion criteria. Individuals with body mass index (BMI) greater than 19.1 and less than 34.9 were included. Before starting, the subjects were informed about the procedures and signed a free and informed consent form, as approved by the local Research Ethics Committee of School of Physical Education and Sports (CAAE:32287614.4.0000.5391). After the consent to participate in the research, the participants were divided into two different groups: long-HIIT and short-HIIT, with total of 6 weeks. The participants performed 18 training sessions, performed 3 times a week, for 6 weeks. All anthropometric and aerobic fitness measures were conducted before and after the intervention.

\section{Sample}

Twenty previously untrained women ( $25 \pm 6$ years), participated in this study. They were instructed not to change their eating routine and not to perform any regular physical activity program during the study. The characteristics of the sample are shown in Table 1.

\section{Measurements}

\section{Anthropometric measurements}

For the analysis of body composition, it was used the BMI, waist-hip ratio and skinfold thicknesses. For the BMI, the body mass were collected through a portable digital scale (Tanita, Tokyo, Japan) and the height through a wooden stadiometer. The calculation of BMI was made by dividing body mass $(\mathrm{kg})$ by the square of the height $(\mathrm{cm})$. For the measurement of the perimeters (waist, hip, arm, thorax, thigh, and leg) a fiberglass measuring tape was used. The waist-hip ratio was calculated by the ratio of the perimeter of the waist by hip girth. The corrected thigh circumference proposed by Harrison et al. (1988) was calculated in order to isolate muscle mass. For the skinfold measurements (indirect method for the prediction of body fat), an adipometer (CESCORF, Porto Alegre, Rio Grande do Sul, Brazil) was used to measure 3 times on rotation basis the following skinfold thicknesses: triceps, subscapular, pectoral, suprailiac, abdominal, thigh, and leg. The median of the three measurements was utilized. The estimation of body density and fat percentage was performed from the generalized equation of Jackson et al. (1980) of four skinfolds, being tricipital, suprailiac, abdominal and thigh and equation of Siri (1961), respectively. From the percentage of fat, the FM was estimated. The fat free mass (FFM) was calculated by subtracting the FM from the total body mass. 


\section{Aerobic power estimation}

On the same day of the anthropometric measurements, a submaximal test proposed by Astrand and Ryhming (1954) was performed on a cycle ergometer to estimate the $\mathrm{VO}_{2 \max }$. This test estimates the $\mathrm{VO}_{2 \max }$ through the heart rate response with the use of progressive loads at submaximal levels. The estimation of $\mathrm{VO}_{2 \max }$ was performed using the modified Astrand-Ryhming nomogram.

\section{Food intake}

It was requested to the participants to fill out a food diary before and after the intervention, with the time and amount of food ingested in three nonconsecutive days of the week, one of them being necessarily the weekend. The analysis was made from the app FatSecret (Secret Industries Pty Ltd., Caulfield North, VIC, Australia) (Subar et al., 2010).

\section{Perspective measurements}

A visual analogue scale (VAS) was applied to evaluate the hunger perception immediately after each training session. The scale ranged from 1 to 10 , with 1 corresponding to no hunger perception and to 10 the maximum hunger perception. It has been shown that VAS is highly effective in predicting food intake and has high reproducibility (Flint et al., 2000).

After each session, RPE was collected using the CR-10 scale adapted from Borg (Grant et al., 1999), where the subject reported on a scale of 1 to 10 the RPE, with 1 (little or no exertion) and 10 (maximum perceived exertion).

\section{Training protocols}

The two groups (short- and long-HIIT) underwent a 3-min warm-up prior to the session and maintained a no-load activity in the final 3 min of the session; the warm-up load was $60 \%$ of maximal HR. Each session consisted of 15 min of effort through HIIE with work: rest ratio equal to 2:1 and loads referring to $90 \%$ of the HRmax (in the work phase) and $60 \%$ of the HRmax (in the recovery phase). The training equalization was done using the training volume. The duration of the series were different in each group: long-HIIT performed 1 min of work with a $30 \mathrm{sec}$ of recovery, with total of 15 series, while short-HIIT performed $20 \mathrm{sec}$ of work with $10 \mathrm{sec}$ of recovery, with total of 45 series. To determine the maximum HR, the formula of Tanaka et al. (2001) prediction by age was used.

At each training session, the HR values were measured (Polar Electro Oy, Vantaa, Finland) and from this information the load adjustment was made weekly, so that the percentage of the proposed HR was reached. The cadence was maintained at approximately 60 rotations per minute in both groups, allowing load adjustment (kilopounds).

Table 1. Estimated aerobic power, anthropometry, and energy intake at baseline and after 6 weeks in women submitted to long (1 min:30 sec) or short (20 sec:10 sec) high-intensity interval training protocols

\begin{tabular}{|c|c|c|c|c|}
\hline \multirow{2}{*}{ Variable } & \multicolumn{2}{|c|}{ Pretraining } & \multicolumn{2}{|c|}{ Posttraining } \\
\hline & Long $(n=10)$ & Short $(n=10)$ & Long $(n=10)$ & Short $(n=10)$ \\
\hline Body mass (kg) & $66.9 \pm 10.9$ & $69.0 \pm 14.1$ & $66.3 \pm 11.3$ & $68.7 \pm 13.8$ \\
\hline Body mass index $\left(\mathrm{kg} / \mathrm{m}^{2}\right)$ & $25.4 \pm 3.9$ & $26.0 \pm 4.4$ & $25.2 \pm 4.0$ & $25.9 \pm 4.31$ \\
\hline $\mathrm{FFM}(\mathrm{kg})^{\mathrm{a})}$ & $37.0 \pm 7.1$ & $37.9 \pm 9.6$ & $39.5 \pm 6.1$ & $40.2 \pm 9.7$ \\
\hline $\mathrm{FM}(\mathrm{kg})^{\mathrm{a})}$ & $20.4 \pm 7.5$ & $24.1 \pm 9.5$ & $18.8 \pm 7.3$ & $22.1 \pm 8.9$ \\
\hline Relative FM $(\%)^{a)}$ & $29.4 \pm 6.2$ & $34.0 \pm 5.7$ & $26.8 \pm 6.0$ & $31.1 \pm 5.8$ \\
\hline$\Sigma 7$ skinfolds $(\mathrm{mm})^{\text {a) }}$ & $169.6 \pm 56.9$ & $204.1 \pm 9.6$ & $142.9 \pm 50.8$ & $181.3 \pm 49.3$ \\
\hline \multicolumn{5}{|l|}{ Circumference $(\mathrm{cm})$} \\
\hline Waistal & $75.3 \pm 8.3$ & $80.0 \pm 12.3$ & $73.7 \pm 9.9$ & $78.5 \pm 10.6$ \\
\hline Hip & $100 \pm 9.8$ & $105.9 \pm 11.2$ & $99.5 \pm 10.6$ & $104.6 \pm 11.4$ \\
\hline Ratio waist/hipal & $0.75 \pm 0.05$ & $0.75 \pm 0.05$ & $0.74 \pm 0.04$ & $0.74 \pm 0.05$ \\
\hline Thigh circumference corrected (cm) & $46.0 \pm 4.8$ & $43.3 \pm 4.2$ & $47.6 \pm 3.5$ & $46.5 \pm 3.0$ \\
\hline $\mathrm{VO}_{2 \max }(\mathrm{mL} / \mathrm{kg} / \mathrm{min})^{\mathrm{a})}$ & $27.9 \pm 6.0$ & $33.9 \pm 8.5$ & $36.2 \pm 9.9$ & $41.5 \pm 11.6$ \\
\hline Energy intake (kcal) & $1,444 \pm 556$ & $1,586 \pm 444$ & $1,546 \pm 411$ & $1,668 \pm 520$ \\
\hline
\end{tabular}

Values are presented as mean \pm standard deviation.

Long, long-high-intensity interval training; Short, short-high-intensity interval training; FFM, fat free mass; $\mathrm{FM}$, fat mass; $\mathrm{VO}_{2 m a x}$ maximal oxygen consumption estimated. ${ }^{a}$ Difference between pretraining and posttraing $(P<0.05)$. 


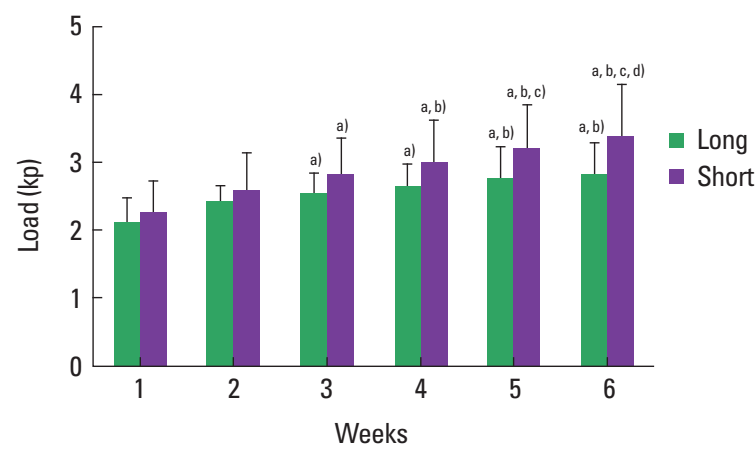

Fig. 1. Load across training sessions in the different training groups (values are mean and standard deviation). kp, kilopounds; Long, long-high-intensity interval training; Short, short-high-intensity interval training. ${ }^{a}$ Values greater than first week $(P<0.05)$. ${ }^{b}$ Values greater than second week $(P<0.05)$. ${ }^{\circ}$ Values greater than third week $(P<0.05)$. Short: ${ }^{d}$ values greater than fourth week $(P<0.05)$.

\section{Statistics}

All variables were analyzed before and after intervention, except for hunger perception, RPE and load which were measured each session. It was also calculated mean values for each week. The descriptive statistical analysis was made via mean and standard deviation calculations. For the inferential analysis to determinate the pre and post intervention changes and the differences between the groups, a two-way (group and moment) analysis of variance with repeated measures was used. When detected a significant difference, the Bonferroni test was used as post hoc. For the variables that were modified with time, the effect size and/or the modification percentage was calculated to verify the magnitude of the intervention effect for each group.

\section{RESULTS}

There was no statistically significant difference between groups at the beginning of the training program. The comparative analyzes are presented in Table 1 .

There was no significant difference or interaction between groups and moments in relation to body mass, BMI, FFM, FM, relative $\mathrm{FM}$, circumferences of wait, hip, thigh, waist hip ratio, $\mathrm{VO}_{2 \max }$, and energy intake.

There was a significant reduction in relative FM between the moments in both groups $(F[1,18]=40.10, P<0.001$; partial $\left.\eta^{2}=0.690\right)$. As expected, the same result was found in FM with significant reduction after the intervention $(F[1,18]=43.76$, $P<0.001$, partial $\left.\eta^{2}=0.647\right)$. The same pattern was found when evaluating the sum of skinfolds between moments $(F[1$, $18]=37.13, P<0.001$, partial $\left.\eta^{2}=0.673\right)$. Since the FFM is calcu-

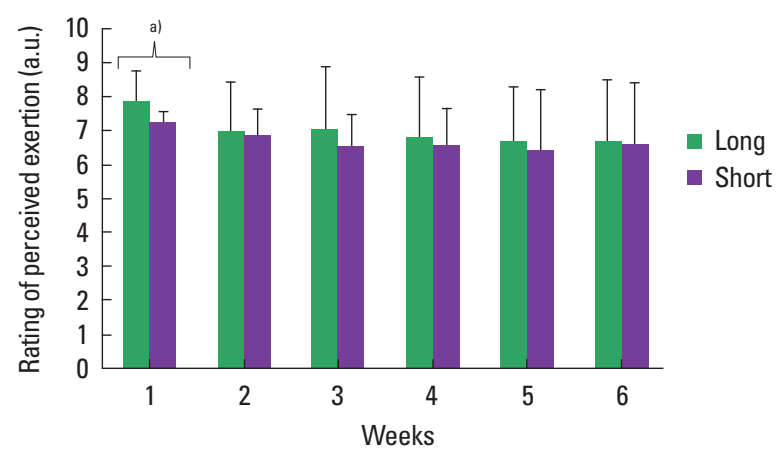

Fig. 2. Rating of perceived exertion across training sessions in the different training groups (values are mean and standard deviation). a.u., arbitrary unit; Long, long-high-intensity interval training; Short, short-high-intensity interval training. ${ }^{\text {a }}$ Values greater than fifth and sixth weeks for both groups $(P<0.05)$.

lated by subtracting FM from the total body mass, a significant increase was found after the intervention $(F[1,18]=25.61, P<$ 0.001 , partial $\eta^{2}=0.587$ ).

There was a reduction in waist circumference after the intervention $\left(F[1,18]=9.36, P=0.006\right.$, partial $\left.\eta^{2}=0.262\right)$, the same response was not found for hip circumference $(F[1,18]=1.30$, $P=0.269$, partial $\eta^{2}=0.067$ ), as a result there was a reduction in waist hip ratio $\left(F[1,18]=6.41, P=0.021\right.$, partial $\left.\eta^{2}=0.262\right)$.

There was an increase in the corrected circumference of the thigh after the intervention $(F[1,18]=19.90, P<0.001$, partial $\left.\eta^{2}=0.525\right)$ and also an increase in $\mathrm{VO}_{2 \max }$ estimated between the moments $\left(F[1,18]=37.57, P<0.001\right.$, partial $\left.\eta^{2}=0.688\right)$.

Once the training prescription was performed through $\mathrm{HR}$, the load was adjusted according to the individual response of each subject. In this way, there was an increase in the load during the sessions for both groups $(F[17,306]=38.40, P<0.001$, partial $\left.\eta^{2}=0.680\right)$ (Fig. 1). Even though there was no difference between the groups $\left(F[17,306]=2.38, P=0.141\right.$, partial $\left.\eta^{2}=0.366\right)$, there was interaction between the group and time $(F[17,306]=2.28$, $P=0.003$, partial $\eta^{2}=0.113$ ), in short protocol until the fourth week there was a significant increase of load, while the load on the long protocol did not increase significantly after the second week. Despite the increase of load, there was a decrease in the RPE (Fig. 2) throughout the training sessions $(F[17,306]=2.79, P<0.001$, partial $\left.\eta^{2}=0.134\right)$. The absence of difference in the RPE reported by the groups $\left(F[17,306]=0.32, P=0.581\right.$, partial $\left.\eta^{2}=0.020\right)$, without interaction between groups $(F[17,306]=0.71, P=0.796$, partial $\eta^{2}=0.037$ ), coincide with the load results. For the hunger perception, measured immediately after each training session, there was no difference between the groups $(F[17,306]=4.24$, $P=0.054$, partial $\left.\eta^{2}=0.172\right)$, time $(F[17,306]=0.82, P=0.674$, 
partial $\left.\eta^{2}=0.043\right)$, or interaction $(F[17,306]=0.91, P=0.560$, partial $\eta^{2}=0.048$ ).

\section{DISCUSSION}

The main find of this study was that both short and long HIIT resulted in reduction in $\mathrm{FM}$, waist circumference, waist hip ratio and on the increase of FFM, thigh circumference, load and $\mathrm{VO}_{2 \max }$ estimated.

Several studies have evaluated the exercise response and body composition in women with similar results, regarding the decrease of delta percentage fat, the magnitude of the decrease was different for each study. In this study we found a decrease of $9 \%$ for long-HIIT and $8.5 \%$ for short-HIIT, without any statistical difference between them. Using protocols characterized as RST $(60 \times 8$-sec all-out with recovery of $12 \mathrm{sec})$ Airin et al. (2014) and Trapp et al. (2008) found similar results (-6.8\% and $-8 \%$, respectively), with sample composed by eutrophic and overweight women. Hazell et al. (2014) and Higgins et al. (2016) used a SIT protocol ( 4 to $7 \times 30$-sec all-out with $4 \mathrm{~min}$ of recovery) for 6 weeks and found a decrease of $8 \%$ and $2.37 \%$ respectively. Although both studies used the same protocol and showed a reduction in fat percentage, the amplitude of the decline was greater in the Hazell et al. (2014), with a sample composed of eutrophic and overweight active women, where the training sessions were performed in cycle ergometer, while Higgins et al. (2016) had the sample formed by inactive women with overweight and obesity and performed the sessions in the treadmill. These factors can influence the results. Racil et al. (2013) found a reduction of 7.8\% in a sample composed by obese adolescents using a short-HIIT protocol ( 2 series of $6 \times 30 \mathrm{sec}$ a $100 \%-110 \%$ maximal aerobic speed (MAS) with recovery interval of $30 \mathrm{sec}$ a $50 \%$ MAS with 4 min of recovery between the series) performed for 12 weeks.

Simultaneously to the reduction of fat percentage, Airin et al. (2014), Hazell et al. (2014), Racil et al. (2013), and Trapp et al. (2008) showed a reduction in body mass $(-1.41 \%,-0.82 \%$, $-3.83 \%$, and $-2.37 \%$, respectively). The studies that presented greater weight reduction had a longer period of intervention, as Racil et al. (2013) for 12 weeks and Trapp et al. (2008) for 15 weeks. This study did not find significant difference concerning body mass, as well as Higgins et al. (2016).

Although the mechanisms responsible for weight loss and fat percentage decrease are still unclear, one of the hypotheses is the acute reduction on appetite (hormones, hunger perception and energy intake per se). However, the acute response studies aiming to investigate the effect of exercise intensity in women failed in detect reduction of energy intake due exercise intensity (Panissa et al., 2016; Sim et al., 2015). In this study, there was no difference between the groups or throughout the sessions in the hunger perception collected immediately after each session, as there was no difference in preintervention and postintervention on food intake, similarly to the findings of studies that evaluated food intake (Hazell et al., 2014; Higgins et al., 2016; Racil et al., 2013; Trapp et al., 2008). These results suggest that, although there was no change in the perception of hunger or food intake, we have to consider the energy expenditure promoted in all sessions, which can change the energy balance on training days and probably affect body composition if longer training period is conducted.

The aforementioned studies, which duration of 6 weeks, also found an increase in lean body mass (1.15\%) (Airin et al., 2014) and FFM (1.30\%) (Hazell et al., 2014). These data support the data of this study (long-HIIT, 7\% and short-HIIT, 6\%). The increase in FFM suggests muscle hypertrophy, although Mora-Rodríguez et al. (2016), using a specific technique (biopsies), showed that, after four months of HIIT ( 4 sets of 4 min at 90\% HRmax) there was a reduction of body and FM and an increase of FFM of leg. However, it was due to an increase of water and glycogen, while a reduction of $11 \%$ of protein was found.

As it is already clear, different HIIE protocols have different acute responses, which can induce different chronicle adaptations. The previous training status, body composition, type of ergometer, and the type of equipment used to measure body composition can affect distinctly short- and long-term responses.

As found in the study by Price and Moss (2007), we expected to find higher RPE scores in the group that performed the interval exercise protocol with longer effort duration when compared to the interval exercise protocol with shorter effort duration. Even though there was no difference between groups, once the prescription was established according to the HR, the groups responded similarly in terms of RPE. In addition to that, throughout the sessions the RPE decreased, showing an adaptation to the protocols, even though the load was increased to keep the HR response constant during the training sessions. The load increase during training period was more frequent in short-HIIT than long-HIIT and must be explored by future studies in order to identify this impact on parameters related to physical fitness. This suggests dissociation between HR, RPE and load during HIIE sessions and should be considered when prescribing HIIE. The present results suggest that both types of training (short- or long-HIIT) can modify the body composition of adult women without changes in 
food intake. Since the two protocols presented similar results, the possibility of modifying the duration of effort with maintenance of the work:rest relationship is interesting in a training program with the objective of giving the practitioner new challenges, avoiding the monotony and increasing adherence. In addition, the short-HIIT generated greater impact on the increase of the load and this can be considered in the training prescription.

\section{CONFLICT OF INTEREST}

No potential conflict of interest relevant to this article was reported.

\section{ACKNOWLEDGMENTS}

We would like to thank the Institutional Program of Scientific Initiation CNPQ (100595/2015-4). Valéria Leme Gonçalves Panissa is supported by FAPESP (2015/11302-3) and Emerson Franchini is supported by a grant from CNPq (302242/2014-7). We would like to thank the Cardiovascular Rehabilitation and Exercise Physiology - InCor - USP.

\section{REFERENCES}

Airin S, Linoby A, Zaki MSM, Baki H, Sariman H, Esham B, Azam MZM, Mohamed MN. The effects of high-intensity interval training and continuous training on weight loss and body composition in overweight females. In: Adnan R, Ismail S, Sulaiman N, editors. Proceedings of the International Colloquium on Sports Science, Exercise, Engineering and Technology; 2014 April 7-9; Penang, Malaysia. Singapore: Springer Singapore; 2014: p. 401-409.

Astrand PO, Ryhming I. A nomogram for calculation of aerobic capacity (physical fitness) from pulse rate during sub-maximal work. J Appl Physiol 1954;7:218-221.

Blair SN, Church TS. The fitness, obesity, and health equation: is physical activity the common denominator? JAMA 2004;292:1232-1234.

Buchheit M, Laursen PB. High-intensity interval training, solutions to the programming puzzle: Part I: cardiopulmonary emphasis. Sports Med 2013;43:313-338

Ekkekakis P. Let them roam free? Physiological and psychological evidence for the potential of self-selected exercise intensity in public health. Sports Med 2009;39:857-888.

Flint A, Raben A, Blundell JE, Astrup A. Reproducibility, power and validity of visual analogue scales in assessment of appetite sensations in single test meal studies. Int J Obes Relat Metab Disord 2000;24:38-48.
Frazão DT, de Farias Junior LF, Dantas TC, Krinski K, Elsangedy HM, Prestes J, Hardcastle SJ, Costa EC. Feeling of pleasure to high-intensity interval exercise is dependent of the number of work bouts and physical activity status. PLoS One 2016;11:e0152752.

Garber CE, Blissmer B, Deschenes MR, Franklin BA, Lamonte MJ, Lee IM, Nieman DC, Swain DP; American College of Sports Medicine. American College of Sports Medicine position stand. Quantity and quality of exercise for developing and maintaining cardiorespiratory, musculoskeletal, and neuromotor fitness in apparently healthy adults: guidance for prescribing exercise. Med Sci Sports Exerc 2011;43:13341359.

Gillen JB, Martin BJ, MacInnis MJ, Skelly LE, Tarnopolsky MA, Gibala MJ. Twelve weeks of sprint interval training improves indices of cardiometabolic health similar to traditional endurance training despite a five-fold lower exercise volume and time commitment. PLoS One 2016;11:e0154075.

Gist NH, Freese EC, Cureton KJ. Comparison of responses to two high-intensity intermittent exercise protocols. J Strength Cond Res 2014;28: 3033-3040.

Grant S, Aitchison T, Henderson E, Christie J, Zare S, McMurray J, Dargie $\mathrm{H}$. A comparison of the reproducibility and the sensitivity to change of visual analogue scales, Borg scales, and Likert scales in normal subjects during submaximal exercise. Chest 1999;116:1208-1217.

Harrison GG, Buskirk ER, Carter JEL, Johnston FE, Lohman TG, Pollock ML, Roche AF, Wilmore J. Skinfold thicknesses and measurement technique. In: Lohman TG, Roche AF, Martorell R, editors. Anthropometric standardization reference manual. Champaign: Human Kinetics Books; 1988. p.55-70.

Hauser C, Benetti M, Rebelo FP. Weight loss strategies. Rev Bras Cineantropom Desempenho Hum 2004;6:72-81.

Hazell TJ, Hamilton CD, Olver TD, Lemon PW. Running sprint interval training induces fat loss in women. Appl Physiol Nutr Metab 2014; 39:944-950.

Higgins S, Fedewa MV, Hathaway ED, Schmidt MD, Evans EM. Sprint interval and moderate-intensity cycling training differentially affect adiposity and aerobic capacity in overweight young-adult women. Appl Physiol Nutr Metab 2016;41:1177-1183.

Islam H, Townsend LK, Hazell TJ. Modified sprint interval training protocols. Part I. Physiological responses. Appl Physiol Nutr Metab 2017; 42:339-346.

Jackson AS, Pollock ML, Ward A. Generalized equations for predicting body density of women. Med Sci Sports Exerc 1980;12:175-181.

Macpherson RE, Hazell TJ, Olver TD, Paterson DH, Lemon PW. Run sprint interval training improves aerobic performance but not maximal cardiac output. Med Sci Sports Exerc 2011;43:115-122. 
Mora-Rodríguez R, Sanchez-Roncero A, Fernández-Elías VE, Guadalupe-Grau A, Ortega JF, Dela F, Helge JW. Aerobic exercise training increases muscle water content in obese middle-age men. Med Sci Sports Exerc 2016;48:822-828.

Nalcakan GR. The effects of sprint interval vs. continuous endurance training on physiological and metabolic adaptations in young healthy adults. J Hum Kinet 2014;44:97-109.

Panissa VL, Alves ED, Salerno GP, Franchini E, Takito MY. Can shortterm high-intensity intermittent training reduce adiposity? Sport Sci Health 2016;12:99-104.

Price M, Moss P. The effects of work: rest duration on physiological and perceptual responses during intermittent exercise and performance. J Sports Sci 2007;25:1613-1621.

Racil G, Ben Ounis O, Hammouda O, Kallel A, Zouhal H, Chamari K, Amri M. Effects of high vs. moderate exercise intensity during interval training on lipids and adiponectin levels in obese young females. Eur J Appl Physiol 2013;113:2531-2540.

Rankin JW. Effective diet and exercise interventions to improve body composition in obese individuals. Am J Lifestyle Med 2015;9:48-62.

Sim AY, Wallman KE, Fairchild TJ, Guelfi KJ. Effects of High-Intensity Intermittent Exercise Training on Appetite Regulation. Med Sci Sports Exerc 2015;47:2441-2449.

Siri WE. Body composition from fluid spaces and density: analysis of methods. In: Tecnhiques for measuring body composition. Quarter- master and research and engeneering center; 1959 Jan 22-23; Natick, Massachusetts. Washington: National academy of Sciences - National Research Council; 1961. p. 223-244.

Smith-Ryan AE. Enjoyment of high-intensity interval training in an overweight/obese cohort: a short report. Clin Physiol Funct Imaging 2017;37:89-93.

Subar AF, Crafts J, Zimmerman TP, Wilson M, Mittl B, Islam NG, McNutt S, Potischman N, Buday R, Hull SG, Baranowski T, Guenther PM, Willis G, Tapia R, Thompson FE. Assessment of the accuracy of portion size reports using computer-based food photographs aids in the development of an automated self-administered 24-hour recall. J Am Diet Assoc 2010;110:55-64.

Tanaka H, Monahan KD, Seals DR. Age-predicted maximal heart rate revisited. J Am Coll Cardiol 2001;37:153-156.

Trapp EG, Chisholm DJ, Freund J, Boutcher SH. The effects of high-intensity intermittent exercise training on fat loss and fasting insulin levels of young women. Int J Obes (Lond) 2008;32:684-691.

Tremblay A, Simoneau JA, Bouchard C. Impact of exercise intensity on body fatness and skeletal muscle metabolism. Metabolism 1994;43: 814-818.

Tschakert G, Kroepfl J, Mueller A, Moser O, Groeschl W, Hofmann P. How to regulate the acute physiological response to "aerobic" high-intensity interval exercise. J Sports Sci Med 2015;14:29-36. 